\title{
Minor-class splicing occurs in the nucleus of the Xenopus oocyte
}

\author{
KYLE FRIEND, ${ }^{1}$ NIKOLAY G. KOLEV, ${ }^{1}$ MEI-DI SHU, and JOAN A. STEITZ \\ Department of Molecular Biophysics and Biochemistry, Howard Hughes Medical Institute, Yale University, \\ New Haven, Connecticut 06511, USA
}

\begin{abstract}
A small fraction of premessenger RNA introns in certain eukaryotes is excised by the minor spliceosome, which contains lowabundance small nuclear ribonucleoproteins (snRNPs). Recently, it was suggested that minor-class snRNPs are localized to and function in the cytoplasm of vertebrate cells. To test whether U12-type splicing occurs in the cytoplasm of Xenopus oocytes, we performed microinjections of the well-characterized P120 minor-class splicing substrate into the nucleus or into the cytoplasm. Our results demonstrate that accurate splicing of this U12-dependent intron occurs exclusively in the nuclear compartment of the oocyte, where U12 and U6atac snRNPs are primarily localized. We further demonstrate that splicing of both a major-class and a minor-class intron is inhibited after nuclear envelope breakdown during meiosis.
\end{abstract}

Keywords: minor-class splicing; U12 snRNA; Xenopus oocyte

\section{INTRODUCTION}

In eukaryotes, many mRNAs are synthesized as pre-mRNAs with introns that are spliced out of the final, translated transcript. Splicing is conducted by a large ribonucleoprotein complex, the spliceosome, and the major-class spliceosome includes U1, U2, U4, U5, and U6 snRNPs. More recently, it was discovered that vertebrates, plants, and many other eukaryotic lineages contain a second spliceosome that removes a distinct class of introns with different consensus sequences at the $5^{\prime}$ splice site, $3^{\prime}$ splice site, and intronic branchpoint (Hall and Padgett 1994; Russell et al. 2006; Sheth et al. 2006). The minor spliceosome is compositionally different from the major spliceosome, containing U11, U12, U4atac, and U6atac snRNPs, whereas U5 snRNP is shared by both major and minor spliceosomes (Hall and Padgett 1996; Tarn and Steitz 1996a,b). While the majority of introns are spliced by the major spliceosome, $\sim 1 / 300$ human pre-mRNA introns are processed by the minor spliceosome (Sheth et al. 2006).

\footnotetext{
${ }^{1}$ These authors contributed equally to this work.

Reprint requests to: Joan A. Steitz, Department of Molecular Biophysics and Biochemistry, Howard Hughes Medical Institute, Yale University, New Haven, CT 06511, USA; e-mail: joan.steitz@yale.edu; fax: (203) 624-8213.

Article published online ahead of print. Article and publication date are at http://www.rnajournal.org/cgi/doi/10.1261/rna.1119708.
}

Consistent with observations that major-class splicing occurs in the nucleus, evidence has accumulated that minor-class splicing is similarly nuclear. U11 and U12 snRNAs were originally characterized in nuclear RNA pools (Montzka and Steitz 1988), and even before these snRNPs were functionally characterized, U11 and U12 snRNAs were localized to the nucleus of mammalian cells (Matera and Ward 1993). Additionally, minor spliceosome-specific proteins, such as the U11/U12-associated proteins $35 \mathrm{~K}$ and $31 \mathrm{~K}$ localize to the nucleus in both plant and HeLa cells (Lorković et al. 2005; Wang et al. 2007).

However, recent experiments have suggested that minorclass splicing is a cytoplasmic process (König et al. 2007). König et al. reported predominantly cytoplasmic localization of U12 and U6atac snRNAs, as well as minor-class intron-containing pre-mRNAs, in zebrafish tissue and mammalian cells. Furthermore, the authors found that the minor spliceosome is not down-regulated during mitosis, in contrast to observations that major-class splicing is inhibited after nuclear envelope breakdown during the M phase of the cell cycle (Shin and Manley 2002).

Xenopus laevis oocytes have been used extensively to study major-class splicing. The major spliceosome actively splices pre-mRNAs microinjected into oocyte germinal vesicles (GVs, the nuclei of unfertilized oocytes) (Green et al. 1983). In particular, snRNA levels are efficiently knocked down in oocytes microinjected with complementary DNA oligonucleotides due to endogenous RNase $\mathrm{H}$ 
(Pan and Prives 1988). Although U11, U4atac, and U6atac snRNAs have not yet been characterized in Xenopus, U12 snRNA has (Yu et al. 1996) and is expressed in Xenopus oocytes (Friend et al. 2007). In addition, oocytes matured by the addition of progesterone undergo GV breakdown (Smith 1989) and are therefore an excellent model system to examine splicing inhibition/activity in the absence of the nuclear membrane (Nurse 1990). Here, we have asked whether minor-class splicing is nuclear or cytoplasmic in the Xenopus oocyte and whether the minor spliceosome is active after nuclear breakdown.

\section{RESULTS AND DISCUSSION}

To test whether minor-class splicing can occur in the cytoplasm of Xenopus oocytes, we used the P120 minorclass splicing substrate that has previously been shown to be spliced by the minor spliceosome in HeLa nuclear extract (Tarn and Steitz 1996b). Capped RNA was transcribed in vitro and injected into either the GV (nucleus) or cytoplasm of immature, stage VI oocytes. After incubation, oocytes were manually dissected into nuclear and cytoplasmic fractions, and radiolabeled RNA was separated by PAGE to resolve the splicing products (Fig. 1A). Both the spliced RNA and excised intron-lariat accumulated only in the nucleus three hours after the P120 splicing substrate was microinjected into GVs (Fig. 1A, lanes 2 and 3). No products were observed in either compartment when the substrate was injected into the cytoplasm (Fig. 1A, lanes 4 and 5). Although it has been reported that minor-class splicing is not inhibited by nuclear breakdown (König et al. 2007), we were unable to observe detectable splicing of the P120 substrate after microinjection into oocytes matured with progesterone (Fig. 1A, lane 6).

To verify that the P120 substrate was spliced by the minor spliceosome in the oocyte, Xenopus U12 snRNA (Yu et al. 1996) was knocked down by microinjecting an antisense DNA oligonucleotide into the cytoplasm of oocytes. Endogenous RNase $\mathrm{H}$ activity present within the oocyte then destroys U12 snRNA hybridized to this oligonucleotide. Subsequently, radiolabeled P120 substrate was microinjected into the GVs of either mock-treated or U12-depleted oocytes and incubated. Nuclear RNA was isolated and resolved by denaturing PAGE (Fig. 1B), indicating that splicing of the P120 substrate (Fig. 1B, lane 1) was inhibited after U12 snRNA depletion (Fig. 1B, lane 2). Control RT-PCR reactions and Northern blots were performed to demonstrate efficient knockdown of U12 snRNA, but not disruption of U1, U2, or U6 snRNA levels (Fig. 1C).

To demonstrate that the P120 splicing substrate was indeed spliced by the minor spliceosome, the spliced product was amplified by RT-PCR and sequenced. The sequence of the junction between the $5^{\prime}$ and $3^{\prime}$ splice sites confirms accurate splicing by the minor spliceosome (Fig. 1D).
To address whether major-class splicing was inhibited after GV breakdown, we microinjected the adenovirus standard major-class splicing substrate (Yu et al. 1998) into immature and mature oocytes. Consistent with the observation that extracts derived from mitotic cells do not perform major-class splicing (Shin and Manley 2002), we similarly observed that adenovirus standard substrate splicing is inhibited after GV breakdown (Fig. 1E).

The splicing results we have obtained for Xenopus oocytes do not agree with the proposed localization of both U12 and U6atac snRNAs to the cytoplasm in NIH 3T3 cells and zebrafish tissue (König et al. 2007). We therefore directly examined the location of both U12 and U6atac snRNAs in Xenopus oocytes. The sequence of Xenopus U6atac snRNA has not previously been reported, but we identified this highly conserved snRNA in both Xenopus tropicalis and Xenopus laevis (Supplemental Fig. S1). GVs were manually separated from the oocyte cytoplasm, and RNA was prepared from equivalent numbers of oocytes. RT-PCR indicated that U12 snRNA is exclusively present in the GV fraction from oocytes whereas U6atac snRNA is enriched in the nucleus (Fig. 1F). Since GV volume is only about 1/25th the volume of the oocyte (Gall 2006), the concentrations of U12 and even of U6atac snRNAs are more than an order of magnitude higher in the nucleus than in the cytoplasm. As controls, both U2 and U6 snRNAs were highly enriched in the nuclear fraction, whereas 5.8S rRNA was found exclusively in the cytoplasmic fraction. Unfortunately, U11 and U4atac snRNAs have not been reported for Xenopus laevis, nor were we able to identify these snRNAs by searching the most recent Xenopus genome databases.

Xenopus oocytes are considered an excellent experimental system to examine the subcellular compartmentalization of biological processes (Yang et al. 1992). We find that minor-class splicing occurs exclusively within the nucleus of the Xenopus oocyte. Splicing was detected only when the P120 substrate was microinjected into the GV and not into the cytoplasm of intact oocytes. After GV injection, no spliced product appeared in the cytoplasm, further arguing that minor-class splicing is completed in the nucleus. This splicing depended on intact U12 snRNA, which is clearly localized to the oocyte GV in cellular fractionation experiments. Even for U6atac snRNA, the concentration is significantly higher in the nuclear fraction of the oocyte. Finally, we observed that minor-class splicing was inhibited in oocytes that had undergone GV breakdown, a process similar to nuclear breakdown during mitosis (Nurse 1990).

Our data extend previous reports that minor spliceosome components localize to the nucleus (Matera and Ward 1993; Lorković et al. 2005; Wang et al. 2007) and that, as is the case for major-class splicing, nuclear extract contains all the components necessary to perform minor-class splicing (Tarn and Steitz 1996b). Furthermore, minor-class introns participate in exon definition with 


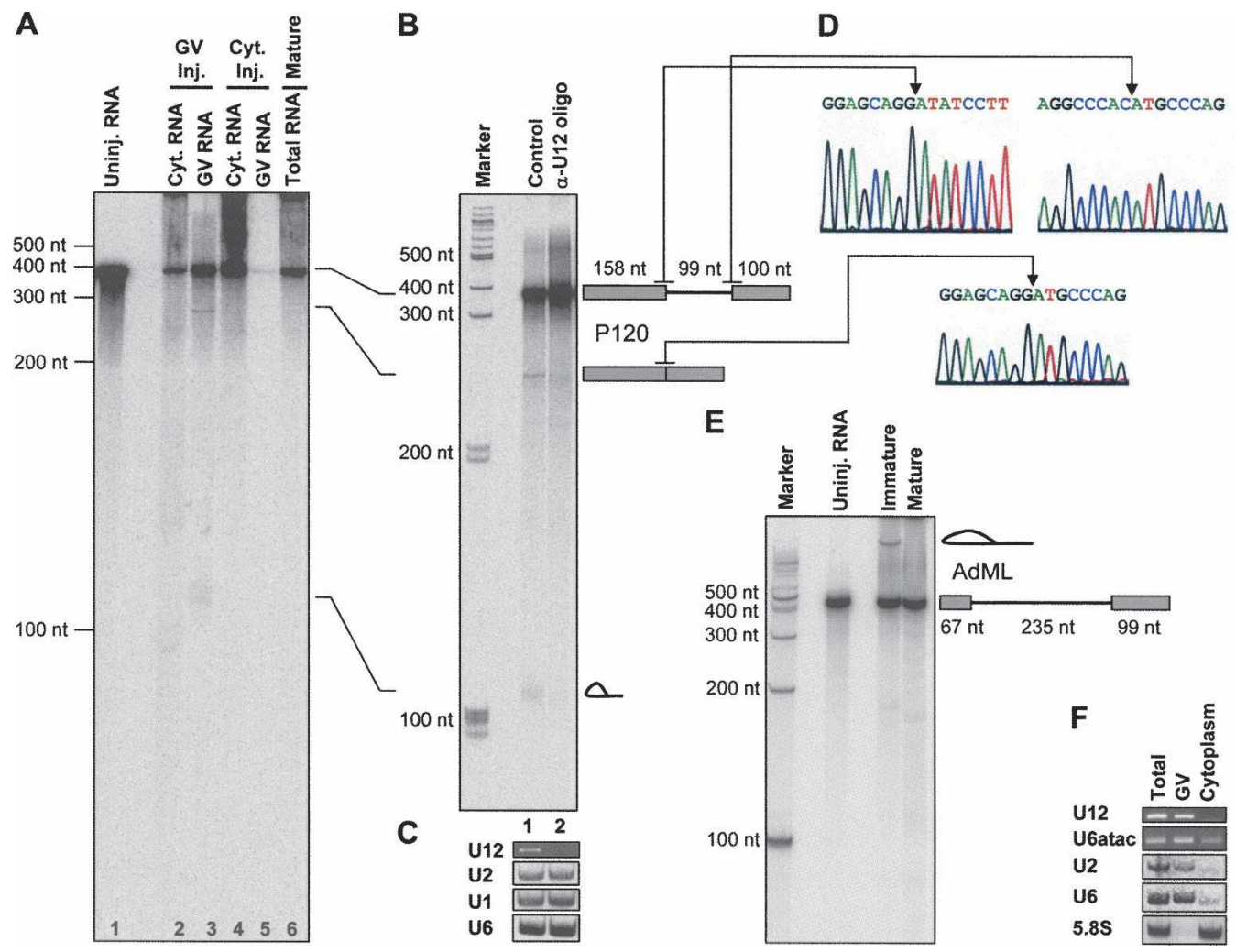

FIGURE 1. (A) $\mathrm{m}^{7} \mathrm{G}$-capped P120 substrate RNA was transcribed with T7 RNA polymerase in the presence of $\left[\alpha^{3}{ }^{32} \mathrm{P}\right] \mathrm{UTP}$ and gel-purified (Tarn and Steitz 1996b; Frilander and Steitz 1999). Stage VI Xenopus laevis oocytes were injected with $9.2 \mathrm{~nL}$ containing $8.3 \times 10^{3} \mathrm{cpm}(\sim 2.5$ fmol) P120 RNA and $20 \mathrm{mg} / \mathrm{mL}$ blue dextran, which serves as a marker for successful nuclear injection, either through the animal pole (GV injection) or the vegetal pole (cytoplasmic injection). Alternatively, mature oocytes were obtained by treatment with progesterone until germinal vesicle breakdown was visualized as a white spot at the animal pole (Smith 1989). All injected oocytes were incubated for $3 \mathrm{~h}$ before manually separating the GV from the cytoplasm. RNA was isolated from these fractions or from mature oocytes with TRIZOL reagent (Invitrogen). Eight oocytes were selected and combined for each sample. RNA (cytoplasmic, GV, or total mature) was separated on a denaturing $8 \%$ polyacrylamide gel. Analysis with ImageQuant software (Molecular Dynamics) of short (overnight) and long (four days, shown) exposures of the dried gel indicated that the efficiency of splicing was $\sim 1 \%$ and that $\sim 0.2 \%$ splicing could have been detected above background in the cytoplasm. (B) Oocytes were injected in the cytoplasm with water (control) or $92 \mathrm{ng}$ oligodeoxyribonucleotide 5'-GTTGTTATTTTCCTTACTC$3^{\prime}$ complementary to U12 snRNA ( $\alpha$-U12 oligo) 15 h prior to injection of the P120 RNA substrate into the nucleus. After 3 h incubation, GV RNA was prepared as in A from 10 oocytes for each sample. RNA was resolved on a denaturing 7\% polyacrylamide gel. The positions of the P120 substrate, the spliced product (inhibited by $\sim 80 \%$ by ImageQuant analysis), and the intron-lariat are indicated on the right. (C) The same GV RNA samples were used to verify the knockdown of U12 snRNA by RT-PCR (upper panel) as described previously (Friend et al. 2007), whereas levels of U2 snRNA, U1 snRNA, and U6 snRNA were assessed by Northern blotting (lower panels; probe sequences contained in supplemental information). Lane 1 is the control; lane 2 shows U12 knockdown. (D) Sequencing of the P120 spliced product to verify the accuracy of minorclass splicing in the oocyte system. Both the splicing substrate and spliced product were amplified by nested RT-PCR from GV RNA and sequenced (primer sequences contained in Supplemental Materials). To amplify the spliced product, the pre-mRNA was first digested using RNase $\mathrm{H}$ and the DNA oligonucleotide 5'-CTCCTAACTCTTCACTCTGC-3' complementary to the P120 intron. The arrows mark the 5' and 3' splice sites of the substrate and the splice junction of the product. (E) Major-class splicing is inhibited upon GV breakdown. Adenovirus standard splicing substrate (Yu et al. 1998) was microinjected into GVs of immature or the animal pole of mature oocytes $\left(9.0 \times 10^{3} \mathrm{cpm}, \sim 2.5 \mathrm{fmol}\right)$ and incubated for $30 \mathrm{~min}$. Total RNA was isolated from nine oocytes per sample and separated on a denaturing $10 \%$ polyacrylamide gel. The unspliced precursor and intron-lariat splicing product are schematized. $(F)$ U12 and U6atac snRNAs are highly enriched in the nucleus of Xenopus oocytes. After manual separation of GVs and cytoplasm from five oocytes, RNA was prepared from these samples as well as from five undissected oocytes (total RNA). U12 and U6atac snRNAs were detected by RT-PCR, while U2 snRNA, U6 snRNA, and 5.8S rRNA were visualized by Northern blotting (probe sequences contained in Supplemental Materials).

major-class introns, which are certainly spliced in the nucleus (Wu and Krainer 1996). Finally, it has been observed that ribosomes translate mRNAs as they transit into the cytoplasm (Mehlin et al. 1992), so the minor spliceosome would have to compete with translating ribosomes in order to perform (or complete) splicing in the cytoplasm. In addition to our findings, a recent report demonstrates that U11, U12, U4atac, and U6atac snRNAs as well as $\mathrm{U} 11-59 \mathrm{~K}, \mathrm{U} 11-35 \mathrm{~K}$, and $\mathrm{U} 11-25 \mathrm{~K}$ proteins are enriched in the nucleus (Pessa et al. 2008). These data argue strongly that the minor spliceosome is localized in the nucleus and functions there. 


\section{SUPPLEMENTAL DATA}

Supplemental material can be found at http://www.rnajournal.org.

\section{ACKNOWLEDGMENTS}

We thank D. Mishler for providing templates for P120 and AdML transcription and A. Miccinello for editorial assistance. This work was supported by grant R01GM026154 from the NIGMS. The content is solely the responsibility of the authors and does not necessarily represent the official views of the NIGMS or the NIH. J.A.S. is an investigator of the Howard Hughes Medical Institute.

Received April 3, 2008; accepted April 21, 2008.

\section{REFERENCES}

Friend, K., Lovejoy, A.F., and Steitz, J.A. 2007. U2 snRNP binds intronless histone pre-mRNAs to facilitate U7-snRNP-dependent $3^{\prime}$-end formation. Mol. Cell 28: 240-252.

Frilander, M.J. and Steitz, J.A. 1999. Initial recognition of U12dependent introns requires both $\mathrm{U} 11 / 5^{\prime}$ splice-site and U12/ branchpoint interactions. Genes \& Dev. 13: 851-863.

Gall, J.G. 2006. Exporting actin. Nat. Cell Biol. 8: 205-207.

Green, M.R., Maniatis, T., and Melton, D.A. 1983. Human $\beta$-globin pre-mRNA synthesized in vitro is accurately spliced in Xenopus oocyte nuclei. Cell 32: 681-694.

Hall, S.L. and Padgett, R.A. 1994. Conserved sequences in a class of rare eukaryotic nuclear introns with nonconsensus splice sites. J. Mol. Biol. 239: 357-365.

Hall, S.L. and Padgett, R.A. 1996. Requirement of U12 snRNA for in vivo splicing of a minor class of eukaryotic nuclear pre-mRNA introns. Science 271: 1716-1718.

König, H., Matter, N., Bader, R., Thiele, W., and Müller, F. 2007. Splicing segregation: The minor spliceosome acts outside the nucleus and controls cell proliferation. Cell 131: 718-729.

Lorković, Z.J., Lehner, R., Forstner, C., and Barta, A. 2005. Evolutionary conservation of minor U12-type spliceosome between plants and humans. RNA 11: 1095-1107.

Matera, A.G. and Ward, D.C. 1993. Nucleoplasmic organization of small nuclear ribonucleoproteins in cultured human cells. J. Cell Biol. 121: 715-727.

Mehlin, H., Daneholt, B., and Skoglund, U. 1992. Translocation of a specific premessenger ribonucleoprotein particle through the nuclear pore studied with electron microscope tomography. Cell 69: 605-613.

Montzka, K.A. and Steitz, J.A. 1988. Additional low-abundance human small nuclear ribonucleoproteins: U11, U12, etc. Proc. Natl. Acad. Sci. 85: 8885-8889.

Nurse, P. 1990. Universal control mechanism regulating onset of M-phase. Nature 344: 503-508.

Pan, Z.Q. and Prives, C. 1988. Assembly of functional U1 and U2 human-amphibian hybrid snRNPs in Xenopus laevis oocytes. Science 241: 1328-1331.

Pessa, H.K.J., Will, C.L., Meng, X., Schneider, C., Watkins, N.J., Perälä, N., Mymark, M., Turunen, J.T., Lührmann, R., and Frilander, M.J. 2008. Minor spliceosome components are predominantly localized in the nucleus. Proc. Natl. Acad. Sci. 105: 8655-8660.

Russell, A.G., Charette, J.M., Spencer, D.F., and Gray, M.W. 2006. An early evolutionary origin for the minor spliceosome. Nature 443: 863-866.

Sheth, N., Roca, X., Hastings, M.L., Roeder, T., Krainer, A.R., and Sachidanandam, R. 2006. Comprehensive splice-site analysis using comparative genomics. Nucleic Acids Res. 34: 3955-3967.

Shin, C. and Manley, J.L. 2002. The SR protein SRp38 represses splicing in M phase cells. Cell 111: 407-417.

Smith, L.D. 1989. The induction of oocyte maturation: Transmembrane signaling events and regulation of the cell cycle. Development 107: 685-699.

Tarn, W.Y. and Steitz, J.A. 1996a. Highly diverged U4 and U6 small nuclear RNAs required for splicing rare AT-AC introns. Science 273: $1824-1832$.

Tarn, W.Y. and Steitz, J.A. 1996b. A novel spliceosome containing U11, U12, and U5 snRNPs excises a minor class (AT-AC) intron in vitro. Cell 84: 801-811.

Wang, H., Gao, M.X., Li, L., Wang, B., Hori, N., and Sato, K. 2007. Isolation, expression, and characterization of the human ZCRB1 gene mapped to 12q12. Genomics 89: 59-69.

$\mathrm{Wu}$, Q. and Krainer, A.R. 1996. U1-mediated exon definition interactions between AT-AC and GT-AG introns. Science 274: 1005-1008.

Yang, H., Moss, M.L., Lund, E., and Dahlberg, J.E. 1992. Nuclear processing of the $3^{\prime}$-terminal nucleotides of pre-U1 RNA in Xenopus laevis oocytes. Mol. Cell. Biol. 12: 15531560.

Yu, Y.T., Shu, M.D., and Steitz, J.A. 1998. Modifications of U2 snRNA are required for snRNP assembly and pre-mRNA splicing. EMBO J. 17: 5783-5795.

Yu, Y.T., Tarn, W.Y., Yario, T.A., and Steitz, J.A. 1996. More Sm snRNAs from vertebrate cells. Exp. Cell Res. 229: 276-281. 

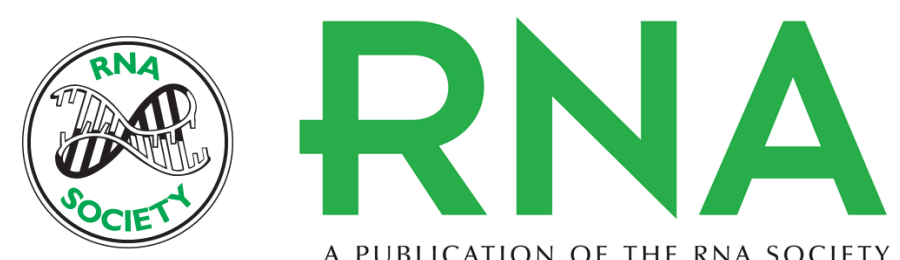

A PUBLICATION OF THE RNA SOCIETY

\section{Minor-class splicing occurs in the nucleus of the Xenopus oocyte}

Kyle Friend, Nikolay G. Kolev, Mei-Di Shu, et al.

RNA 2008 14: 1459-1462

Supplemental
Material http://rnajournal.cshlp.org/content/suppl/2008/06/26/rna.1119708.DC1

References This article cites 25 articles, 12 of which can be accessed free at: http://rnajournal.cshlp.org/content/14/8/1459.full.html\#ref-list-1

License

Email Alerting Receive free email alerts when new articles cite this article - sign up in the box at the Service top right corner of the article or click here. 